\title{
BMJ Open Helpful explanatory models for somatoform symptoms (HERMES): study protocol of a randomised mixed- methods pilot trial
}

\author{
Paul Hüsing (D , ${ }^{1}$ Bernd Löwe, ${ }^{1}$ Tim C Olde Hartman, ${ }^{2}$ Lisbeth Frostholm, ${ }^{3}$ \\ Angelika Weigel ${ }^{1}$
}

To cite: Hüsing $P$, Löwe $B$, Olde Hartman TC, et al. Helpful explanatory models for somatoform symptoms (HERMES): study protocol of a randomised mixedmethods pilot trial. BMJ Open 2021;11:e044244. doi:10.1136/ bmjopen-2020-044244

- Prepublication history for this paper is available online. To view these files, please visit the journal online (http://dx.doi. org/10.1136/bmjopen-2020044244).

Received 27 August 2020 Revised 24 February 2021 Accepted 12 March 2021

\section{Check for updates}

\section{Author(s) (or their} employer(s)) 2021. Re-use permitted under CC BY-NC. No commercial re-use. See rights and permissions. Published by BMJ.

${ }^{1}$ Department of Psychosomatic Medicine and Psychotherapy, University Medical Center Hamburg-Eppendorf, Hamburg, Germany

${ }^{2}$ Department of Primary and Community Care, Radboud University Medical Center, Nijmegen, The Netherlands

${ }^{3}$ Research Clinic for Functional Disorders and Psychosomatics, Aarhus University Hospital, Aarhus, Denmark

Correspondence to

Dr Paul Hüsing;

p.huesing@uke.de

\section{ABSTRACT}

Introduction Persistent somatic symptoms (PSS) are common both in the general population and primary care. They are bothersome in terms of psychological and somatic symptom burden. Health professionals often struggle with communication, as there is a lack of scientifically supported explanatory models for PSS or a focus merely on somatic aspects of the complaints, which both frustrate patients' needs. The objective of the present study is therefore to develop a psychoeducational intervention based on a current evidence-based explanatory model, to examine its feasibility and form the basis for a large-scale randomised controlled trial.

Methods and analysis In a randomised controlled mixed-methods pilot trial, 75 adult psychosomatic outpatients with PSS (duration of symptoms $\geq 6$ months) and accompanying psychological (Somatic Symptom B-Criteria Scale total score $\geq 18$ ) and somatic symptom burden (Patient Health Questionnaire-15 score $>10$ ) and no prior psychosomatic treatment will be eligible. Participants will be presented with either the explanatory model without (intervention group $1, \mathrm{n}=25$ ) or with elements of personalisation (intervention group $2, n=25$ ). Participants in the control group $(n=25)$ will receive information on current PSS guidelines. Participants will be blinded to group assignment and interventions will be shown on tablet computers at the outpatient clinic. After 1 month, qualitative follow-up telephone interviews will be conducted. As primary outcomes, mean changes in psychological and somatic symptom burden will quantitatively be compared between groups, respectively. Behavioural change mechanisms and feasibility of the three interventions will be evaluated using quantitative and qualitative measures.

Ethics and dissemination Ethics approval has been granted by the medical ethics board of the Hamburg Medical Chamber (PV5653). Results from this study will be published in peer-reviewed journals and presented at national and international conferences.

Trial registration number DRKS00018803.

\section{INTRODUCTION}

Persistent somatic symptoms (PSS) are marked by long-lasting (typically more than 6 months) physical afflictions and sensations
Strengths and limitations of this study

- This is the first study to translate a state-of-the-art aetiological model for persistent somatic symptoms (PSS) into an explanatory model that can be used in daily patient care.

- Feedback both from a patient group and an international expert panel was used during the development of the psychoeducational interventions, which employ means of visualisation and personalisation.

- The sample will consist of 75 patients with PSS in three experimental groups in order to ensure sufficient power for the pilot trial, using a mixedmethods approach.

- Limitations include the diverse target population with heterogeneous symptom profiles, thus restricting the external validity of the results.

- The present study is limited to a brief psychoeducational intervention on a tablet computer without further face-to-face interaction.

and include complaints associated with disorders such as fibromyalgia, irritable bowel syndrome or somatic symptom disorder. ${ }^{1}$ PSS are a common phenomenon both in the general population and medical settings. At the same time, treatment options are still limited and patients are often regarded as 'bothersome' and 'frustrating' to work with. ${ }^{2}$ PSS can affect every organ system and the estimated lifetime prevalence ranges between $5.9 \%$ and $16.3 \%$ in the general population and specialised settings. ${ }^{3-5}$ In primary care, approximately $20 \%$ of all consultations are estimated to be due to PSS. ${ }^{67}$ PSS are often accompanied by psychological symptoms at cognitive, emotional and behavioural level. ${ }^{8-11}$ The term PSS in the context of this study summarises PSS that reach diagnostic threshold according to current diagnostic classifications (eg, somatoform disorders), ${ }^{1213}$ somatic symptom disorder (Diagnostic and Statistical Manual of Mental Disorders, Fifth 
Edition/DSM-5), ${ }^{14}$ bodily distress disorder (International Classification of Diseases 11th Revision). ${ }^{15}$

The impairments caused by PSS affect individuals in terms of a significantly reduced quality of life ${ }^{4}$ and the healthcare system in terms of excessive healthcare costs due to medical consultations and diagnostic procedures. ${ }^{16}$ Despite their individual and socioeconomic relevance, the majority of affected patients receive no treatment according to guidelines, ${ }^{17-19}$ thus enabling a chronic course of disease. ${ }^{20}$ Additionally, there is a considerable delay between PSS symptom onset and the start of an adequate treatment, that is, psychological interventions. ${ }^{21}{ }^{22}$ Further, treatment options for PSS are limited and multidisciplinary treatment as well as nonpharmacological interventions show only moderate effect sizes. ${ }^{23}$ Thus, there is a great need to improve early treatment in PSS.

Early interventions usually include psychoeducational material. While there is evidence for the general usefulness of psychoeducational interventions for medically unexplained symptoms (MUS) and functional disorders, ${ }^{24-26}$ the lack of evidence-based explanatory models has been identified as one central barrier in the early treatment of PSS. ${ }^{27}$ A systematic search of MUS literature identified nine different explanatory models for PSS. ${ }^{28}$ These explanatory models were based on somatosensory amplification, sensitisation, sensitivity, immune system sensitisation, endocrine dysregulation, the signal filter model, the illness behaviour model, autonomous nervous system dysfunction and abnormal proprioception, and also included one meta-model, the cognitive-behavioural therapy model. However, most of these models were only partly based on empirical evidence. In primary care, health professionals often feel insecure about the management of patients with PSS. ${ }^{2}$ They explain the development and maintenance of symptoms only vaguely, and without any references to current aetiological models ${ }^{29-32}$ thereby failing patients' needs for biomedical and tangible explanatory models. ${ }^{29}{ }^{33-35}$ When symptom explanations and treatment are not readily available, medical doctors may rely on a defensive biomedical approach in dealing with the symptoms. ${ }^{19}$ The patients on the other hand may continue to search for the biomedical diagnosis which explains their symptoms, ${ }^{36}$ that is, examinations for shorttime reassurance instead of actively engaging in treatment, for example, with the help of knowledge of illness or self-management strategies. ${ }^{37}$

Recently, a new aetiological model on PSS was suggested by Henningsen et $a l^{38}$ While historically, aetiological models of symptom persistence emphasised bottom-up processes in a biomedical context and further developed into a biopsychosocial understanding, this new model on PSS focuses on top-down processes and conceptualises symptoms as a perceptual dysregulation. It represents a comprehensive biopsychosocial model of PSS and incorporates evidence-based findings, that is, by enhancing existing vulnerability stress models and emphasising the role of the patients' perception and expectations regarding their symptoms. The model has not yet been translated into an explanatory model adapted for the use with patients with PSS. As the authors conclude, 'a major challenge remains to develop metaphors and motivational techniques to convince patients to go along with these strategies and modify the patient's illness belief' ${ }^{38}$

In order to make explanatory models more accessible, provided information needs to be clearly formulated, articulated in an understandable way and avoid unnecessary and distracting content. ${ }^{39}$ Providing feedback on PSS is a peculiar challenge, as patients might feel disbelieved by their doctors in case a biomedical focus is left. ${ }^{27}$ Most patients have distinct preferences for how they would choose to receive health information, including the possible use of media. Visual health information is becoming more widely used to communicate information about health and illness to patients. When used in an intervention, it can improve patients' illness understanding and may ameliorate health behaviour such as adherence to treatment. ${ }^{40}$ As illness understanding requires a certain level of abstract thinking, visual information may make these intangible processes easier to understand. ${ }^{41-43}$ Additionally, visual information is often easier to attend to and to be remembered compared with more traditional forms of information. ${ }^{44}$

Apart from visualisation, the provided information should be considered as relevant by the patient, thus increasing the likelihood of it being used. To increase individual relevance, the information has to address needs or fears of the individual, ${ }^{45}$ should be adjusted to the patients' level of communication ${ }^{46}$ and give patients an active choice in the selection of the content. ${ }^{47}$ Such a person-centred, or personalised, approach has been shown to be of value for patients with long-term conditions such as PSS. ${ }^{48}$

In conclusion, explanatory models for PSS are thus far not based on current aetiological models, existing knowledge is not available in a way understandable for patients and their practitioners and not designed to suit individual patient needs. However, a current state-of-the-art aetiological model combined with the use of active visualisation and personalisation might be a valuable approach for both patients and their practitioners to communicate explanatory models for PSS.

\section{OBJECTIVES}

The primary aim of the HERMES pilot study (full title: Helpful explanatory models in somatoform symptoms) is to explore the feasibility and the impact of an intervention formed on an evidence-based explanatory model which can be used as a state-of-the-art early psychoeducational intervention to improve the physical and psychological symptom burden in patients with PSS. We thus translated the aetiological model on PSS suggested by Henningsen et al into an explanatory model that fits both patients' and practitioners' needs, ${ }^{35} 394950$ and that employs means of visualisation. Development of this explanatory model 
was sustained by the use of feedback both by a patient group and an expert panel, and feasibility of the model will be tested in patients with PSS and their practitioners. Therefore, this study will serve as proof-of-concept and feasibility study to form the basis for a randomised controlled study, using explanatory models as a starting point to improve early treatment for patients with PSS. ${ }^{51}$ Additionally, the present study will allow an estimation of randomised controlled trial (RCT) eligibility as well as recruitment and attrition rates. Last, the present study will examine the effect of this newly developed explanatory model both with and without elements of personalised medicine on psychological and physical symptom burden.

\section{Study hypotheses}

The primary hypothesis is that the presentation of explanatory models for PSS in both experimental conditions results in significantly greater changes in psychological and physical symptom burden between baseline and 1-month follow-up compared with the control group. It is further explored whether the additional provision of choice regarding patient information leads to significantly greater changes in psychological and somatic symptom burden compared with an explanatory model without choice. Exploratory analyses will shed light on whether the presentation of explanatory models for PSS in the experimental conditions leads to a significantly greater improvements in quality of life between baseline and 1-month follow-up compared with the control condition.

\section{METHODS AND ANALYSIS}

\section{Study design}

A pilot RCT was designed to evaluate the influence of evidence-based explanatory models for PSS on somatic and psychological symptom burden. Data will be collected at baseline and after 1 month. Independent variables will be operationalised through the experimental conditions. At baseline, participants will be randomised into one of three experimental conditions: explanatory model without personalised choice versus explanatory model with personalised choice versus generic PSS information (control group). Primary dependent variables are psychological and physical symptom burden (figure 1).

\section{Study procedures}

Inclusion and exclusion criteria

Adult patients aged $\geq 18$ years with PSS (duration $\geq 6$ months) and a sum score of $\geq 18$ in the Somatic Symptom B-Criteria Scale (SSD-12) ${ }^{52}$ and $\geq 10$ in the Patient Health Questionnaire-15 (PHQ-15) ${ }^{53}$ and thus at risk for somatic symptom disorder ${ }^{54}$ will be included. Written informed consent will be collected.

Exclusion criteria are insufficient knowledge of the German language, current or previous psychotherapeutic treatment of PSS, an acute need for treatment due to other comorbid psychological disorders, and the possibility of acute self-harm or endangerment of others.

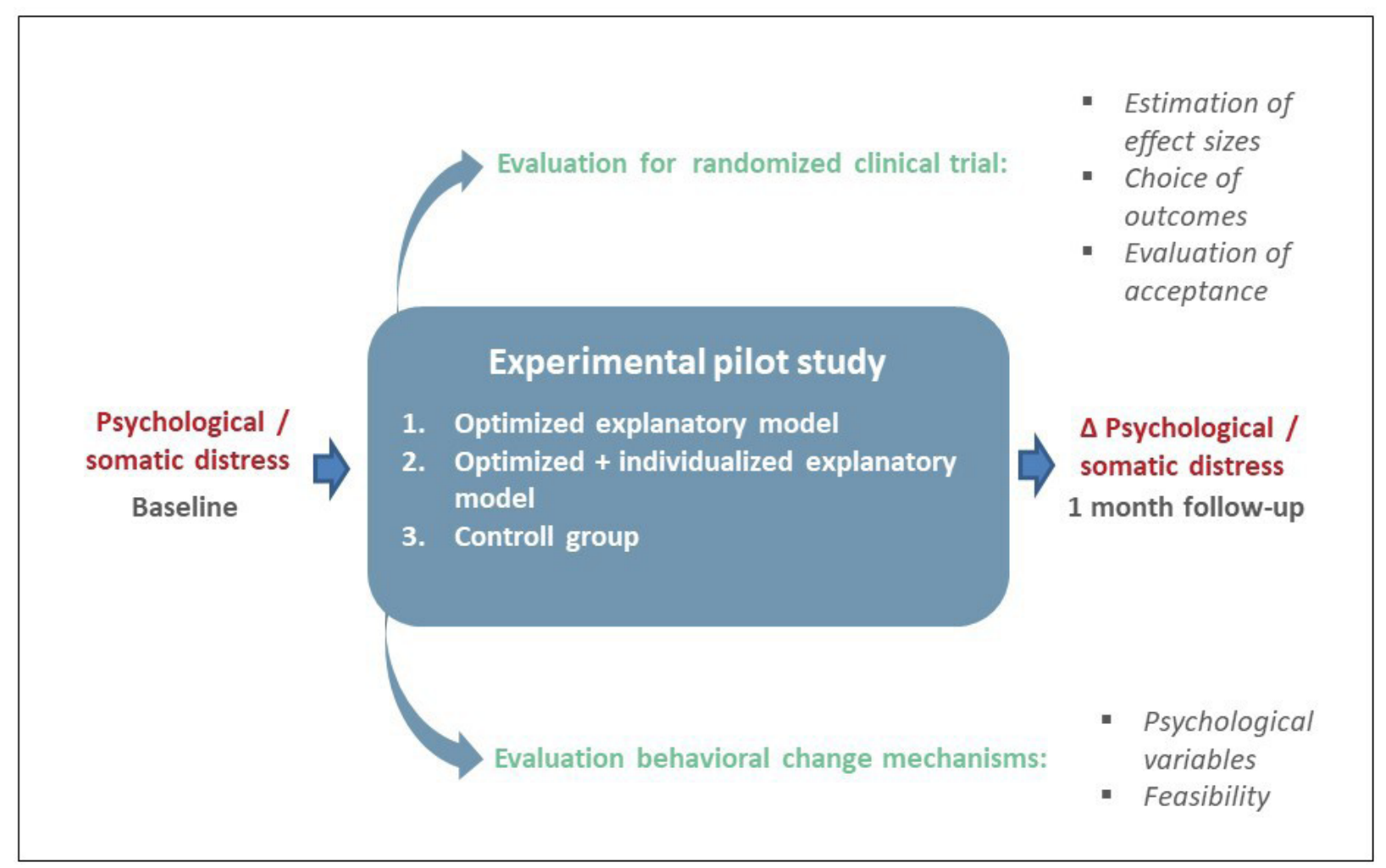

Figure 1 Rationale of the HERMES Study. 


\section{Recruitment}

Recruitment will take place in the psychosomatic outpatient clinic of the Department of Psychosomatic Medicine and Psychotherapy at the University Medical Center Hamburg-Eppendorf, Germany. The clinic is specialised in patients with depression, anxiety disorders, eating disorders and somatoform complaints. Patients are usually referred to the clinic after examinations by both the general practitioner (GP) and specialists. In the clinic, patients undergo a thorough psychosomatic evaluation (self-report measures and clinical interview) and receive assistance in the choice and finding of adequate treatment, as well as first clinical interventions. Eligible patients for the study will be verbally informed about the study by the attending clinician after consultation. Information on the study and consent will be provided in written form. In case of willingness to participate, a member of the study team will explain the course of the experiment in detail, answer questions and collect the written consent. If the patient declines to participate despite eligibility, the clinician will document age, sex and the reasons for refusal to participate. Follow-up interviews will be conducted via telephone 1 month after baseline.

\section{Patient and public involvement}

Patients and the public were not involved in the initial design of the study. During development of the intervention, feedback from a patient group will be employed in the design of the interventions.

\section{Randomisation}

Randomisation will take place after patients are included, based on a random number system and an urn randomisation. Patients will be blinded regarding their group assignment.

\section{INTERVENTION GROUPS}

In order to ensure internal validity, frequency, duration and performance will be kept on an equal level within all three experimental conditions. Demonstration of the respective explanatory model will take place immediately after randomisation. Duration of all three interventions will be approximately $15 \mathrm{~min}$ each and the digital content will be presented on a tablet computer.

\section{Development and design of the interventions}

The explanatory model was designed around the information contained in the aetiological model by Henningsen $e t$ $a l .{ }^{38}$ In a first step, the aetiological model was summarised in a written script with the use of lay language. Then the script was enriched by the application of evidence-based feedback strategies. ${ }^{39}$ Examples of feedback strategies include the use of examples and metaphors, validation, the opportunity for comparisons with other affected patients and prompting statements. The script was then visualised according to means of active visualisation with two different designs, one containing stock photo images and the other using clip art. Information from the script was set to sound by a semiprofessional synchroniser and implemented in the digital presentation. Both versions were then presented to a feedback group of patients with PSS from a group therapy with focus on psychological distress primarily linked to physical complaints. After receiving feedback, the visual content and the script were adjusted accordingly, forming the first draft of the explanatory model. Based on this draft, all three experimental conditions (explanatory model without personalised choice vs explanatory model with personalised choice vs generic PSS information) were designed. These interventions were then shown to an expert panel consisting of two members (LF and TCOH) from a European network on PSS (EURONET-SOMA). ${ }^{1155}$ These experienced clinicians and researchers gave their feedback to the initial script and after an elaborated revision of the material approved the final versions of the three digital experimental interventions, which were then rendered and adjusted to the use on tablet computers (for a process summary, see figure 2).

\section{First experimental condition}

The first experimental condition includes the aetiological model of PSS by Henningsen $e t a l^{88}$ translated into an explanatory model in a language understandable for both patients and healthcare professionals. Illness perceptions, somatosensory amplification and strategies to avoid symptom-related fears will be addressed within the intervention. In order to further optimise the model, phrases from the vernacular and short summaries will be used. ${ }^{35}$ Stigma of mental illness is tried to be minimalised. ${ }^{39}$ No personalised information will be implemented in the intervention.

\section{Second experimental condition}

In the second experimental condition, an element of personal choice will be added to the initial explanatory model. Though personalisation is generally a highly complex and heterogeneous matter, we tried to implement it by providing the patient with a choice concerning his or her individual need regarding maintaining factors of PSS. The first part of the explanatory model will thus contain information equivalent to the first intervention group. However, after the first part the participant will be asked which psychological mechanism is the most relevant for him or her personally: illness perceptions, somatosensory amplification and strategies to avoid symptom-related anxiety. These three aspects were chosen for individualisation based on their specific relevance for the healthcare sector. ${ }^{56-58}$ The second part of the intervention will then address the specific information accordingly. If the participant chooses neither of the factors, the information without choice from the first experimental condition will be shown.

\section{Control condition}

The control condition will be close to treatment as usual, that is, it will neither contain an explanatory model of 


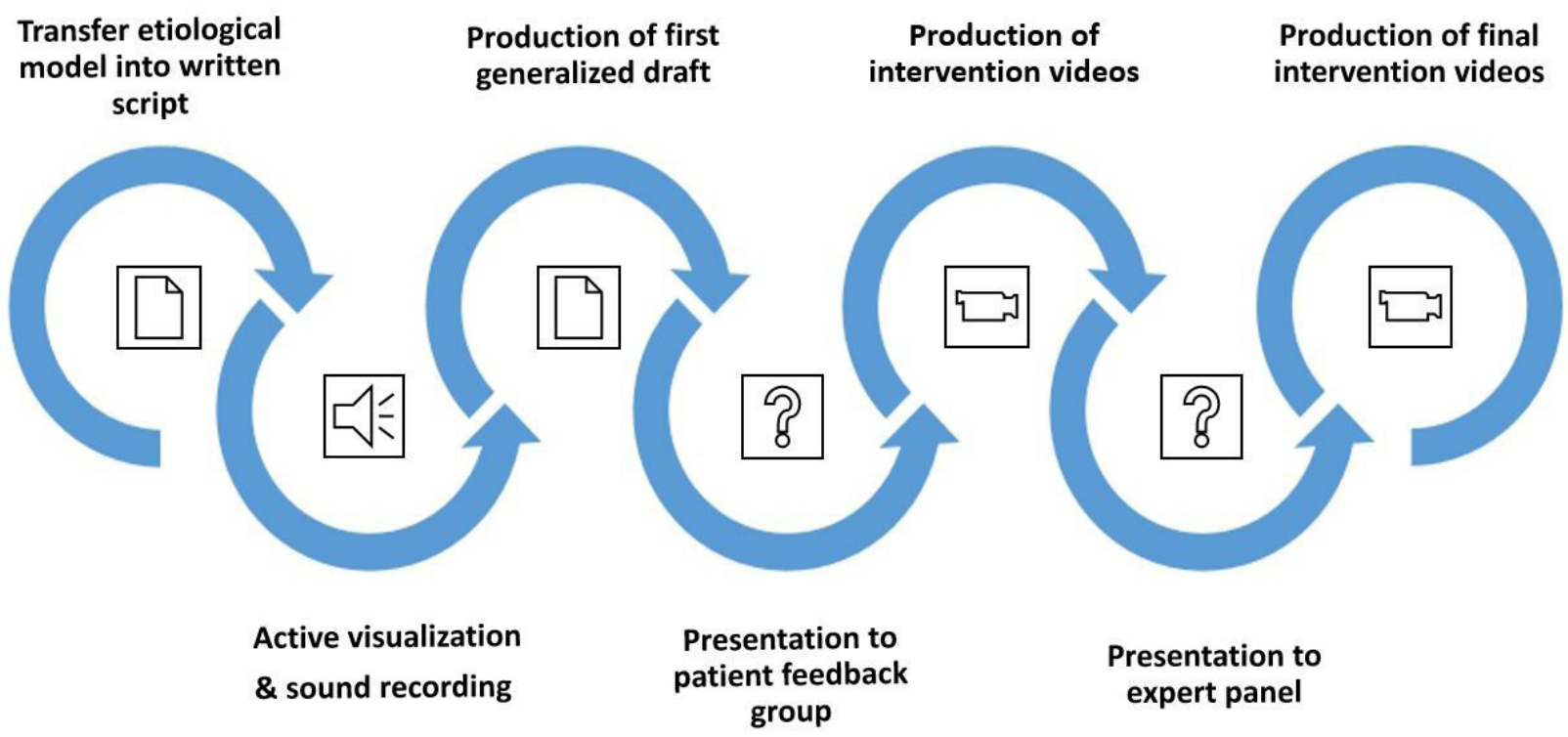

Figure 2 Development process of the video interventions.

PSS, nor maintaining factors, nor personalised information. Participants will instead receive information based on current guidelines for the treatment of PSS. ${ }^{59}$ The information addresses four levels in the treatment of PSS: namely, initial primary care, extended primary care, multimodal treatment and further psychosocial aspects.

\section{VARIABLES AND INSTRUMENTS}

Participants of all three experimental conditions will complete questionnaires at baseline and at 1-month follow-up. An overview of all instruments employed at baseline and follow-up is provided in table 1 .

\section{Acceptance and feasibility of the interventions}

Participation and attrition rates and numerical rating scales on individual acceptance of the intervention and probability to recommend the interventions to family members with PSS will be used to quantitatively estimate acceptance and feasibility of the interventions. Additionally, qualitative aspects of acceptance in terms of applicability of the interventions for individual symptoms and satisfaction of needs will be measured using semistructured interviews. ${ }^{33-35}$ Expectations with regard to the individual course of treatment, positive and negative aspects of the explanatory model, issues of comprehension and general feedback will also be inquired, respectively. At 1-month follow-up, participants will be asked which aspects of the interventions they remember and whether the explanatory model had any influence on their behaviour (ie, using the explanatory model when interacting with friends or family or practitioners). Interviews will be transcribed verbatim and qualitatively analysed applying thematic analysis as suggested by Braun and Clarke. $^{60}$

\section{Psychological and somatic symptom burden}

Primary outcomes of the HERMES pilot study are psychological and somatic symptom burden at 1-month follow-up. Psychological distress due to physical symptoms will be measured using the SSD-12. ${ }^{52}$ The SSD-12 was developed to measure the psychological criteria of somatic symptom disorder according to DSM-5. Each of the three psychological subcriteria (cognitive, affective, behavioural) is measured by four items with scores ranging between 0 and 4, resulting in a total of 12 items with a sum score of $0-48$. The SSD-12 has good item characteristics, excellent reliability (Cronbach's $\alpha=0.95),{ }^{52}{ }^{61}$ and its ability to measure change over time has been established. ${ }^{62}$

The PHQ-15 measures somatic symptom burden and has well-established psychometric properties. ${ }^{63}$ Using 15 items, the PHQ-15 assesses the presence and severity of common somatic symptoms within the last 4 weeks. A cutoff score of $\geq 10$ points has been established to identify patients at risk for clinically relevant symptom burden. Its convergent and divergent validity have been tested in several patient samples and in the general population. ${ }^{5363}$

In addition to the SSD-12 and PHQ-15, we also added two numerical Visual Analogue Scales items on subjective impairment in terms of the intensity of bodily symptoms and their interference with daily life activities over the past 7 days, as recommended by the EURONET-SOMA group. ${ }^{64}$

\section{Psychopathological change mechanisms}

Usefulness of the interventions will be measured using an adapted version of the Usefulness Scale for Patient Information Material. ${ }^{65}$ The questionnaire comprises nine items measuring cognitive, emotional and behavioural usefulness of patient material. The Brief Illness Perception Questionnaire ${ }^{66}$ will indicate changes regarding illness perceptions. Its wide use and good psychometric properties could be demonstrated in a review containing 
Table 1 Overview of instruments employed in the HERMES Study

\begin{tabular}{|c|c|c|c|c|}
\hline Construct & Instrument & $\begin{array}{l}\text { Baseline } \\
\text { (T-0)-before } \\
\text { intervention }\end{array}$ & $\begin{array}{l}\text { Baseline } \\
\text { (T-0)-after } \\
\text { intervention }\end{array}$ & $\begin{array}{l}\text { Follow-up ( } \mathrm{T}- \\
\text { 1) }-4 \text { weeks after } \\
\text { baseline }\end{array}$ \\
\hline Somatic symptom burden & PHQ-15 & $x$ & & $x$ \\
\hline Symptom-related psychological distress & SSD-12 & $\mathrm{x}$ & & $\mathrm{x}$ \\
\hline Quality of life & SF-12 & $x$ & & $x$ \\
\hline Depression & PHQ-9 & $x$ & & \\
\hline Anxiety & GAD-7 & $x$ & & \\
\hline General physical well-being & VAS & $x$ & $x$ & $x$ \\
\hline Illness perceptions & IPQ-B & $x$ & & \\
\hline Avoidance behaviour & VAS & $\mathrm{x}$ & & $\mathrm{x}$ \\
\hline Control behaviour & VAS & $x$ & & $x$ \\
\hline Attention to symptoms & VAS & $\mathrm{x}$ & & $\mathrm{x}$ \\
\hline Physical distress last 7 days & VAS & $x$ & & $x$ \\
\hline $\begin{array}{l}\text { Symptom influence on daily activities over the last } \\
7 \text { days }\end{array}$ & VAS & $\mathrm{x}$ & & $\mathrm{x}$ \\
\hline Catastrophising attribution & FKG & $x$ & & \\
\hline Vegetative discomfort & FKG & & & \\
\hline Actions related to somatic symptoms & HP & $x$ & & $\mathrm{x}$ \\
\hline Usefulness of patient information material & USE & & $x$ & \\
\hline Previous knowledge of information in intervention & VAS & & $\mathrm{x}$ & \\
\hline Fit of information to individual symptoms & VAS & & $\mathrm{x}$ & \\
\hline Credibility of information in intervention & VAS & & $\mathrm{x}$ & \\
\hline $\begin{array}{l}\text { Influence of intervention on perception of physical } \\
\text { complaints }\end{array}$ & VAS & & $x$ & \\
\hline $\begin{array}{l}\text { Influence of intervention on course of treatment with } \\
\text { GP }\end{array}$ & VAS & & $\mathrm{x}$ & \\
\hline Emotional activation due to intervention & Open questions ${ }^{*}$ & & $\mathrm{X}$ & \\
\hline Improvement of intervention & Open questions ${ }^{*}$ & & $x$ & $x$ \\
\hline Remembered content of intervention & Open questions* & & & $\mathrm{x}$ \\
\hline $\begin{array}{l}\text { Influence of intervention on perception of physical } \\
\text { complaints }\end{array}$ & Open question* & & & $\mathrm{x}$ \\
\hline Influence of intervention on course of treatment & Open question* & & & $\mathrm{x}$ \\
\hline
\end{tabular}

${ }^{*}$ Qualitative measure.

FKG, Fragebogen zu Körper und Gesundheit; GAD-7, General Anxiety Disorder Questionnaire-7; GP, general practitioner; HP, actions according to guidelines; IPQ-B, Brief Illness Perception Questionnaire; PHQ-9, Patient Health Questionnaire-9; PHQ-15, Patient Health Questionnaire-15; SF-12, 12-Item Short Form Health Survey; SSD-12, Somatic Symptom B-Criteria Scale; USE, Usefulness Scale for Patient Information Material; VAS, Visual Analogue Scale.

data from 188 studies. ${ }^{67}$ In order to operationalise the concept of somatosensory amplification, the subscales 'catastrophising attribution' and 'vegetative discomfort' of the Questionnaire on Body and Health (Fragebogen zu Körper und Gesundheit) ${ }^{68}$ will be used. Strategies to avoid symptom-related anxiety will be assessed with Visual Analogue Scales on 'physical inactivity' and 'request of medical diagnosis'. Additionally, patients will be asked about functional and dysfunctional actions regarding their somatic complaints in the previous 2 weeks according to the recommendations in the German guidelines on functional somatic complaints in dichotomous format (yes vs no)..$^{59}$

\section{Quality of life}

Using 12 items, the 12-Item Short Form Health Survey ${ }^{69}$ measures psychological and physical aspects of generic, health-related quality of life. Multiple studies could demonstrate its good psychometric properties. ${ }^{70}$

\section{Sample characterisation}

In order to describe the study sample, sociodemographic information on age, gender, education, cultural 
background and data on symptom onset, healthcare utilisation over the past 4 weeks as well as current capacity to work and medication will be collected via the basic service in the outpatient clinic. Data will also include somatic and mental comorbidities: depression will be measured using the PHQ-9, ${ }^{53} 71$ anxiety via the General Anxiety Disorder Questionnaire-7. ${ }^{72}$ Diagnoses from the spectrum of mental disorders will be extracted from the clinical consultation referral letters.

\section{SAMPLE SIZE/POWER CALCULATION}

To our knowledge, this is the first study examining the influence of explanatory models on PSS and their influence on psychological and somatic symptom burden. Hence, there is no prior knowledge concerning sample size calculations. We thus used power calculation with an estimated drop-out rate of $25 \%$ based on a previous study in the same setting, ${ }^{73}$ resulting in an estimated amount of 25 participants per group (75 patients in total). Since the HERMES Study employs brief interventions, one can estimate a small effect size of $\mathrm{f}<0.4$. In combination with three experimental conditions and a double sided $\alpha$-error chance of $5 \%$, a power of up to $78 \%$ ( $1-\beta$ error chance) can be reached, according to the respective effect size. Power calculations were done using the program PASS (V.15.0.3) and an analysis of covariance (ANCOVA) as statistical measure.

\section{Handling of missing values}

Participants with $\geq 25 \%$ missing baseline scores will not be included in the study. In case of missing data either at baseline or follow-up, cases will be analysed according to intention-to-treat principles if a minimum of $75 \%$ of data are present. Handling of missing data will be adapted to missing data patterns. If patients are not available for the interviews at 1-month follow-up, they will be registered as drop-outs after at least five attempts of being reached via telephone. Reason for drop-out will be recorded and only baseline information will be used for analysis. Systematic differences between participants and drop-outs will be examined using the provided data.

\section{Statistical analyses}

Since normal distribution can be assumed for sum scores of symptom burden (psychological symptom burden=SSD-12, somatic symptom burden $=$ PHQ-15), mean score and SD can be used as descriptive characteristic. For evaluation of the primary hypothesis, namely the positive effect of presenting an explanatory model on both psychological and somatic symptom burden, an ANCOVA with experimental group (explanatory model without vs explanatory model with personalised choice vs generic PSS information) as independent variable and changes in symptom burden at 1-month follow-up as dependent variables $\quad($ psychological $=\Delta$ SSD-12; $\quad$ somatic $=\Delta \mathrm{PHQ}-15)$ will be employed, with covariates being age, gender and symptom burden at baseline. Additionally, post-hoc analysis will be used for global comparison of the three experimental conditions. Results will be reported using adjusted mean differences with corresponding 95\% CIs and $\mathrm{p}$ values. Statistical analysis will be performed using IBM SPSS V.23.0. Mediation analysis will be employed for answering the exploratory question concerning mechanisms of action (perceived usefulness of the intervention, illness perceptions, somatosensory amplification, strategies to avoid symptom-related anxiety, and functional and dysfunctional actions regarding somatic complaints) between explanatory model and changes in psychological and somatic symptom burden at 1-month follow-up, using the SPSS macro PROCESS. ${ }^{74}$ Qualitative analysis will be conducted using thematic analysis according to Braun and Clarke. ${ }^{60}$

\section{Methods to reduce bias}

Selection bias is minimised by the inclusion of a control group and an urn randomisation. Presentation of the interventions will be completely standardised by the use of videos on tablet computers. The videos have been created with the attempt to maximise comparability in terms of length, amount of content and visual representation. Both participants and interviewers at 1-month follow-up will be blinded regarding the experimental condition. As it is not possible to blind the scientific assistant during intervention, follow-up interviews will be conducted by an interviewer blinded with regard to the experimental condition. The rate of participants who refrain from signing an informed consent (ie, non-response rate) will be reported. The full recruitment process will be documented according to Consolidated Standards of Reporting Trials reporting standards. ${ }^{75}$

\section{Study registration}

The study was registered at the German Clinical Trials Register (Deutsches Register Klinischer Studien), ${ }^{76}$ and thereby automatically submitted to the WHO International Clinical Trials Registry Platform.

\section{Ethics and dissemination}

The study procedure was reviewed and approved by the medical ethics board of the Hamburg Medical Chamber (approved on 23 October 2017; PV5653). Results from this study will be published in peer-reviewed journals and presented at national and international conferences. Before participation, patients will receive detailed information about the nature, purpose and possible consequences of the trial. Participants will be required to give written informed consent to participate in the study.

\section{DISCUSSION}

The HERMES pilot study aims at developing an evidencebased and visualised explanatory model for PSS, to estimate effect sizes of its influence on both psychological and somatic symptom burden and to evaluate feasibility and acceptance of the intervention. It is hypothesised that the 
presentation of the explanatory model to patients with PSS will result in significant changes in psychological and somatic symptom burden compared with a control group and further, that an additional personalisation through choice of information will lead to an even greater change. Furthermore, we also will explore whether the explanatory models will foster greater improvements in quality of life between baseline and 1-month follow-up compared with the control condition.

Explanatory models are not only part of psychotherapy/ patient-activating treatments, yet form the basis for an individual understanding of complaints. They might thus for example help GPs in their role as gatekeeper within the health system and should easily be employed in early interventions. ${ }^{37}$ By ensuring a high scientific standard in the development and the content of the intervention, we want to fill the void of evidence-based explanatory models for PSS. While there is a number of useful clinical tools and verbalisations available, the majority is limited to clinical use and has not been reviewed within research. ${ }^{28}$ Additionally, we are to our knowledge the first to systematically evaluate an explanatory model for PSS that has been developed using means of active visualisation and personalisation. ${ }^{414248}$

In spite of the numerous advantages of the planned investigation and the high demand for evidence-based explanatory models for PSS, some limitations have to be considered: Visualisation and wording of an intervention are always bound to a subjective nature, and will thus never appeal to all patients. However, by employing a patient feedback group, we will try to minimalise this effect during development of the intervention and use qualitative data for further improvement after the trial. By employing an animated intervention and tablet computers, we are arguably also at risk of entering 'unfamiliar territory' for the patients when it comes to clinical interventions. However, the technological advancements and user data of the past centuries support this change of therapeutic paradigm rather than restraining from it. ${ }^{77-79}$ From a methodological point of view, we are aware that the employed personalisation will be of limited external value in regard to the extremely heterogeneous group of patients with PSS, and as they considerably differ in terms of patients' history, complaints and attitudes towards (psycho)therapeutic approaches. These limitations notwithstanding, we hope to ensure a first insight of the added value of this patientcentred approach in order to foster both effectiveness and appeal of the explanatory model.

If proven effective, the planned interventions will make an important contribution to the early treatment of PSS. They might thus form the basis for the much needed additional research within this area. Future trials might increasingly incorporate concepts of blended interventions, combining various psychoeducational delivery methods. Our use of tablet computers might represent one possible delivery method. Analysis of our data will further provide insight into whether our choice of personalisation is valid or if for example more complex algorithms or the use of psychometrical data might be more suitable approaches.

Contributors AW conceived the study. AW, PH and BL were involved in the concept and design of the study. PH wrote the draft of this manuscript, TCOH, LF, AW and BL provided valuable revisions. All authors contributed to further writing and approved the final version of the manuscript.

Funding The trial is funded by the Werner-0tto-Stiftung (grant number: $n / a$; principal investigator: AW).

Competing interests None declared.

Patient and public involvement Patients and/or the public were not involved in the design, or conduct, or reporting, or dissemination plans of this research.

Patient consent for publication Not required.

Provenance and peer review Not commissioned; externally peer reviewed.

Open access This is an open access article distributed in accordance with the Creative Commons Attribution Non Commercial (CC BY-NC 4.0) license, which permits others to distribute, remix, adapt, build upon this work non-commercially, and license their derivative works on different terms, provided the original work is properly cited, appropriate credit is given, any changes made indicated, and the use is non-commercial. See: http://creativecommons.org/licenses/by-nc/4.0/.

\section{ORCID iD}

Paul Hüsing http://orcid.org/0000-0002-6235-2699

\section{REFERENCES}

1 Kohlmann S, Löwe B, Shedden-Mora MC. Health care for persistent somatic symptoms across Europe: a qualitative evaluation of the EURONET-SOMA expert discussion. Front Psychiatry 2018;9:646.

2 Hahn SR. Physical symptoms and physician-experienced difficulty in the physician-patient relationship. Ann Intern Med 2001;134:897-904

3 Petersen MW, Schröder A, Jørgensen T, et al. Prevalence of functional somatic syndromes and bodily distress syndrome in the Danish population: the DanFunD study. Scand J Public Health 2020;48:567-76.

4 Hiller W, Rief W, Brähler E. Somatization in the population: from mild bodily misperceptions to disabling symptoms. Soc Psychiatry Psychiatr Epidemiol 2006;41:704-12.

5 Hinz A, Ernst J, Glaesmer H, et al. Frequency of somatic symptoms in the general population: normative values for the patient health Questionnaire-15 (PHQ-15). J Psychosom Res 2017;96:27-31.

6 Haller H, Cramer H, Lauche R. Somatoform disorders and medically unexplained symptoms in primary care. Deutsches Ärzteblatt Int 2015;112:279-87.

7 Steinbrecher N, Koerber S, Frieser D, et al. The prevalence of medically unexplained symptoms in primary care. Psychosomatics 2011;52:263-71.

8 Rief W, Isaac M. The future of somatoform disorders: somatic symptom disorder, bodily distress disorder or functional syndromes? Curr Opin Psychiatry 2014;27:315-9.

9 Creed F, Guthrie E, Fink P. Is there a better term than "Medically unexplained symptoms"? J Psychosom Res 2010;68:5-8.

10 Kroenke K, Sharpe M, Sykes R. Revising the classification of somatoform disorders: key questions and preliminary recommendations. Psychosomatics 2007;48:277-85.

11 Euronet-Soma group. EURONET-SOMA - European initiative to improve research, treatment, communication and collaboration on persistent somatic symptoms across the age span, 2018. Available: https://www.euronet-soma.eu/2018

12 World Health Organization. Icd-10: international statistical classification of deseases and related health problems 2004.

13 American Psychiatric Association. Diagnostic and statistical manual of mental disorders : DSM-IV. Washington, DC: American Psychiatric Association, 2000.

14 American Psychiatric Association. Diagnostic and statistical manual of mental disorders: DSM-5. Washington, DC: American Psychiatric Publication, 2013.

15 World Health Organization. International classification of diseases for mortality and morbidity statistics 11th revision, 2018.

16 Fink $\mathrm{P}$, Ewald $\mathrm{H}$, Jensen J, et al. Screening for somatization and hypochondriasis in primary care and neurological in-patients: a 
seven-item scale for hypochondriasis and somatization. J Psychosom Res 1999;46:261-73.

17 Stone J. Functional neurological disorders: the neurological assessment as treatment. Pract Neurol 2016;16:7-17.

18 Henningsen P. Management of somatic symptom disorder. Dialogues Clin Neurosci 2018;20:23.

19 Ring A, Dowrick C, Humphris G, et al. Do patients with unexplained physical symptoms pressurise general practitioners for somatic treatment? A qualitative study. BMJ 2004;328:1057-60.

20 Hausteiner-Wiehle C, Schaefert R, Sattel H. Never say: it's nothing. Improving care for patients with non-specific, functional and somatoform bodily complaints, 2012.

21 Herzog A, Shedden-Mora MC, Jordan P, et al. Duration of untreated illness in patients with somatoform disorders. J Psychosom Res 2018;107:1-6.

22 Kuby AK, Löwe B, Fabisch AB, et al. Predictors of seeking psychotherapy in primary care patients with high somatic symptom burden. Behav Med 2019;45:231-9.

23 van Dessel N, den Boeft M, van der Wouden JC, et al. Nonpharmacological interventions for somatoform disorders and medically unexplained physical symptoms (MUPS) in adults. Cochrane Database Syst Rev 2014;11:CD011142.

24 Melin EO, Svensson R, Thulesius HO. Psychoeducation against depression, anxiety, alexithymia and fibromyalgia: a pilot study in primary care for patients on sick leave. Scand J Prim Health Care 2018;36:123-33.

25 Martin A, Rauh E, Fichter M, et al. A one-session treatment for patients suffering from medically unexplained symptoms in primary care: a randomized clinical trial. Psychosomatics 2007;48:294-303.

26 Louw A, Diener I, Butler DS, et al. The effect of neuroscience education on pain, disability, anxiety, and stress in chronic musculoskeletal pain. Arch Phys Med Rehabil 2011;92:2041-56.

27 Murray AM, Toussaint A, Althaus A, et al. The challenge of diagnosing non-specific, functional, and somatoform disorders: a systematic review of barriers to diagnosis in primary care. J Psychosom Res 2016;80:1-10.

28 van Ravenzwaaij J, Olde Hartman T, van Ravesteijn $\mathrm{H}$. Explanatory models of medically unexplained symptoms: a qualitative analysis of the literature. Ment Health Fam Med 2010;7:223-31.

29 Dwamena FC, Lyles JS, Frankel RM, et al. In their own words: qualitative study of high-utilising primary care patients with medically unexplained symptoms. BMC Fam Pract 2009;10:67.

30 Salmon P. The potentially somatizing effect of clinical consultation. CNS Spectr 2006;11:190-200.

31 Kleinstäuber M, Witthoft M, Steffanowski A. Pharmacological interventions for somatoform disorders in adults. Cochrane Database Syst Rev 2014;11:CD010628.

32 Burton C. Can we explain medically unexplained symptoms? Fam Pract 2014:31:623-4.

33 Bodenheimer T, Wagner EH, Grumbach K. Improving primary care for patients with chronic illness. JAMA 2002;288:1775-9.

34 Hibbard JH, Greene J, Overton V. Patients with lower activation associated with higher costs; delivery systems should know their patients'scores'. Health Aff 2013;32:216-22.

35 Schaefert R, Hausteiner-Wiehle C, Häuser W, et al. Non-specific, functional, and somatoform bodily complaints. Dtsch Arztebl Int 2012;109:803-13.

36 Assing Hvidt E, Lykkegaard J, Pedersen LB, et al. How is defensive medicine understood and experienced in a primary care setting? A qualitative focus group study among Danish general practitioners. BMJ Open 2017;7:e019851.

37 Aiarzaguena JM, Gaminde I, Clemente I, et al. Explaining medically unexplained symptoms: somatizing patients' responses in primary care. Patient Educ Couns 2013;93:63-72.

38 Henningsen P, Gündel H, Kop WJ, et al. Persistent physical symptoms as perceptual dysregulation: a Neuropsychobehavioral model and its clinical implications. Psychosom Med 2018;80:422-31.

39 Brehaut JC, Colquhoun HL, Eva KW, et al. Practice feedback interventions: 15 suggestions for optimizing effectiveness. Ann Intern Med 2016;164:435-41.

40 Karamanidou C, Weinman J, Horne R. Improving haemodialysis patients' understanding of phosphate-binding medication: a pilot study of a psycho-educational intervention designed to change patients' perceptions of the problem and treatment. $\mathrm{Br} J$ Health Psychol 2008;13:205-14.

41 Jones ASK, Kleinstäuber M, Martin LR, et al. Development and validation of the health visual information preference scale. $\mathrm{Br} J$ Health Psychol 2019;24:593-609.

42 Jones ASK, Petrie KJ. I can see clearly now: using active visualisation to improve adherence to art and PreP. AIDS Behav 2017;21:335-40.
43 Jones ASK, Ellis CJ, Nash M, et al. Using animation to improve recovery from acute coronary syndrome: a randomized trial. Ann Behav Med 2016;50:108-18.

44 Gardner MP, Houston MJ. The effects of verbal and visual components of retail communications. J Retailing 1986.

45 Hollands GJ, Marteau TM. The impact of using visual images of the body within a personalized health risk assessment: an experimental study. Br J Health Psychol 2013;18:263-78.

46 Lühnen J, Albrecht M, Hanßen K, et al. Leitlinie evidenzbasierte Gesundheitsinformation: Einblick in die Methodik Der Entwicklung und Implementierung. Zeitschrift für Evidenz, Fortbildung und Qualität im Gesundheitswesen 2015;109:159-65.

47 Kreuter MW, Wray RJ. Tailored and targeted health communication: strategies for enhancing information relevance. Am J Health Behav 2003;27:S227-32.

48 Burton CD, Entwistle VA, Elliott AM, et al. The value of different aspects of person-centred care: a series of discrete choice experiments in people with long-term conditions. BMJ Open 2017;7:e015689.

49 Salmon P, Peters S, Stanley I. Patients' perceptions of medical explanations for somatisation disorders: qualitative analysis. BMJ 1999;318:372-6.

50 Ivers N, Jamtvedt G, Flottorp S, et al. Audit and feedback: effects on professional practice and healthcare outcomes. Cochrane Database Syst Rev 2012;6:CD000259.

51 Lannin DG, Vogel DL, Brenner RE, et al. Does self-stigma reduce the probability of seeking mental health information? J Couns Psychol 2016;63:351.

52 Toussaint A, Murray AM, Voigt K, et al. Development and validation of the somatic symptom disorder-b criteria scale (SSD-12). Psychosom Med 2016;78:5-12.

53 Kroenke K, Spitzer RL, Williams JBW, et al. The patient health questionnaire somatic, anxiety, and depressive symptom scales: a systematic review. Gen Hosp Psychiatry 2010;32:345-59.

54 Toussaint A, Hüsing P, Kohlmann S, et al. Detecting DSM-5 somatic symptom disorder: criterion validity of the Patient Health Questionnaire-15 (PHQ-15) and the Somatic Symptom Scale-8 (SSS8) in combination with the Somatic Symptom Disorder - B Criteria Scale (SSD-12). Psychol Med 2020;50:324-33.

55 Weigel A, Hüsing P, Kohlmann S, et al. A European research network to improve diagnosis, treatment and care for patients with persistent somatic symptoms: work report of the EURONET-SOMA conference series. J Psychosom Res 2017;97:136-8.

56 Voigt $\mathrm{K}$, Wollburg E, Weinmann $\mathrm{N}$, et al. Predictive validity and clinical utility of DSM-5 somatic symptom disorder - comparison with DSM-IV somatoform disorders and additional criteria for consideration. J Psychosom Res 2012;73:345-50.

57 Rief W, Burton C, Frostholm L, et al. Core outcome domains for clinical trials on somatic symptom disorder, bodily distress disorder, and functional somatic syndromes: European network on somatic symptom disorders recommendations. Psychosom Med 2017;79:1008-15.

58 Martin A, Rief W. Relevance of cognitive and behavioral factors in medically unexplained syndromes and somatoform disorders. Psychiatr Clin North Am 2011;34:565-78.

59 Roenneberg C, Hausteiner-Wiehle C, Henningsen P. Funktionelle Körperbeschwerden: Klinisch relevante Leitlinien-Empfehlungen. PSYCH up2date 2020;14:35-53.

60 Braun V, Clarke V. Using thematic analysis in psychology. Qual Res Psychol 2006;3:77-101.

61 Toussaint $A$, Löwe B, Brähler E, et al. The somatic symptom disorder-B criteria scale (SSD-12): factorial structure, validity and population-based norms. J Psychosom Res 2017;97:9-17.

62 Hüsing P, Bassler M, Löwe $B$, et al. Validity and sensitivity to change of the somatic symptom Disorder-B criteria scale (SSD-12) in a clinical population. Gen Hosp Psychiatry 2018;55:20-6.

63 Kroenke K, Spitzer RL, Williams JBW. The PHQ-15: validity of a new measure for evaluating the severity of somatic symptoms. Psychosom Med 2002;64:258-66.

64 Rief W, Burton C, Frostholm L, et al. Core outcome domains for clinical trials on somatic symptom disorder, bodily distress disorder, and functional somatic syndromes: European network on somatic symptom disorders recommendations. Psychosom Med 2017;79:1008-15.

65 Hölzel LP, Ries Z, Dirmaier J, et al. Usefulness scale for patient information material (USE) - development and psychometric properties. BMC Med Inform Decis Mak 2015;15:34.

66 Broadbent E, Petrie KJ, Main J, et al. The brief illness perception questionnaire. J Psychosom Res 2006;60:631-7. 
67 Broadbent E, Wilkes C, Koschwanez $\mathrm{H}$, et al. A systematic review and meta-analysis of the brief illness perception questionnaire. Psychol Health 2015;30:1361-85.

68 Hiller W, Rief W, Elefant S. Dysfunktionale Kognitionen bei Patienten MIT Somatisierungssyndrom. Zeitschrift für Klinische Psychologie 1997;26:226-34.

69 Jenkinson C, Layte R, Jenkinson D, et al. A shorter form health survey: can the SF-12 replicate results from the SF-36 in longitudinal studies? J Public Health Med 1997;19:179-86.

70 Gandek B, Ware JE, Aaronson NK, et al. Cross-validation of item selection and scoring for the SF-12 health survey in nine countries: results from the IQOLA project. International quality of life assessment. J Clin Epidemiol 1998;51:1171-8.

71 Löwe B, Gräfe K, Zipfel S, et al. Diagnosing ICD-10 depressive episodes: superior criterion validity of the patient health questionnaire. Psychother Psychosom 2004;73:386-90.

72 Spitzer RL, Kroenke K, Williams JBW, et al. A brief measure for assessing generalized anxiety disorder: the GAD-7. Arch Intern Med 2006;166:1092-7.
73 Toussaint A, Murray AM, Voigt K. Development and validation of the somatic symptom disorder-B criteria scale (SSD-12) entwicklung und validierung Der somatic symptom Disorder-B criteria scale (SSD-12). Psychosomatic Med 2016;78:5-12.

74 Hayes AF. Introduction to mediation, moderation, and conditional process analysis: a regression-based approach. Guilford Publications, 2017

75 Moher D, Hopewell S, Schulz KF, et al. CONSORT 2010 explanation and elaboration: updated guidelines for reporting parallel group randomised trials. Int J Surg 2012;10:28-55.

76 DRKS. Deutsches register Klinischer Studien (DRKS). Available: https://www.drks.de2019

77 Lindhiem O, Bennett CB, Rosen D, et al. Mobile technology boosts the effectiveness of psychotherapy and behavioral interventions: a meta-analysis. Behav Modif 2015;39:785-804.

78 Stefanopoulou E, Lewis D, Taylor M, et al. Digitally delivered psychological interventions for anxiety disorders: a comprehensive review. Psychiatr Q 2019;90:197-215.

79 Bucci S, Schwannauer M, Berry N. The digital revolution and its impact on mental health care. Psychol Psychother 2019;92:277-97. 